\title{
Contribution of BHG and PPHG to Overall Hyperglycemia in T2DM Patients Treated with LM25 and LM50: Post Hoc Analysis of a Randomized Crossover Trial
}

Wei Li · Fan Ping · Lingling Xu • Huabing Zhang • Yaxiu Dong •

Hongmei Li • Qi Sun • Yuxiu Li (iD

Received: May 12, 2018 / Published online: June 27, 2018

(C) The Author(s) 2018

\begin{abstract}
Introduction: To investigate the relative contribution rates of basal hyperglycemia (BHG) and postprandial hyperglycemia (PPHG) to overall hyperglycemia in patients with type 2 diabetes mellitus (T2DM) treated with insulin lispro mix 25 and 50 (LM25 and LM50) as evaluated by continuous glucose monitoring (CGM).

Methods: Eighty-one T2DM patients treated with premixed human insulin 70/30 (PHI70/30) were randomly divided into two groups and received a crossover protocol. In the first 16-week stage, one group received LM25 twice daily, the other group received LM50 twice daily. In the second 16-week stage, the two groups exchanged therapeutic regimen.
\end{abstract}

Enhanced digital features To view enhanced digital features for this article go to https://doi.org/10.6084/ m9.figshare.6530726.

W. Li · F. Ping · L. Xu · H. Zhang · Y. Dong ·

Q. Sun · Y. Li (ه)

Department of Endocrinology, Key Laboratory of

Endocrinology of National Health and Family

Planning Commission, Peking Union Medical

College Hospital, Chinese Academy of Medical

Sciences and Peking Union Medical College, Beijing,

China

e-mail: liyx@pumch.cn

H. Li

Department of Endocrinology, China Meitan

General Hospital, Beijing, China
Glycosylated hemoglobin $\left(\mathrm{HbA}_{1 \mathrm{c}}\right)$ measurement and CGM were performed at enrollment and at the end of each treatment stage.

Results: BHG's contribution rate increased with increasing $\mathrm{HbA}_{1 \mathrm{c}}$ (from $34.5 \%$ to $60.8 \%$ ). PPHG's contribution rates in the LM50 regimen were significantly lower than those in LM25 and PHI70/30 regimens at $\mathrm{HbA}_{1 \mathrm{c}}$ levels $<7.5 \%$. Compared with LM50, LM25 shows a significant difference in reducing $\mathrm{HbA}_{1 \mathrm{c}}$ in the subgroup with baseline $\mathrm{HbA}_{1 \mathrm{c}} \geq 8.5 \% \quad\left(\Delta \mathrm{HbA}_{1 \mathrm{c}}\right.$ LM25 vs. LM50 $-0.6 \pm 0.1 \%$ vs. $0.3 \pm 0.1 \%$, $p<0.05)$.

Conclusions: For T2DM patients treated with premixed insulin analogues, postprandial hyperglycemia played a major role in the subgroup of patients with $\mathrm{HbA}_{1 \mathrm{c}}<8.5 \%$, while fasting hyperglycemia became the major contributor to overall hyperglycemia in the subgroup of patients with $\mathrm{HbA}_{1 \mathrm{c}} \geq 8.5 \%$.

Trial Registration: Chinese Clinical Trial Registry Identifier ChiCTR-TTRCC-12002516.

Funding: Lilly Suzhou Pharmaceutical Co., Ltd. (Shanghai Branch, China) and National Key Program of Clinical Science of China (WBYZ2011-873).

Keywords: Insulin lispro; PPHG's contribution rate; Premixed human insulin; Type 2 diabetes mellitus 


\section{INTRODUCTION}

Glycemic control is important for reducing the risk of diabetes-related complications. In some large studies, such as DCCT/EDIC, UKPDS, and its follow-up study, glycosylated hemoglobin $\left(\mathrm{HbA}_{1 \mathrm{c}}\right)$ was used to measure blood glucose levels [1-6]. $\mathrm{HbA}_{1 \mathrm{c}}$ generally reflects the average blood glucose levels in the prior 3 months. However, for real-time assessment of blood glucose levels, $\mathrm{HbA}_{1 \mathrm{c}}$ is not as clearly visualized as checking the blood glucose level directly. Therefore, the relationship between blood glucose and $\mathrm{HbA}_{1 \mathrm{c}}$ has been investigated in many studies.

Monnier et al. published a milestone study that described the relative contribution rates of basal hyperglycemia (BHG) and postprandial hyperglycemia (PPHG) to overall hyperglycemia at different $\mathrm{HbA}_{1 \mathrm{c}}$ levels in subjects receiving no treatment of insulin or acarbose [7]. The study showed that for patients with low $\mathrm{HbA}_{1 \mathrm{c}}$ levels $(<7.3 \%)$, the contribution rate of PPHG to overall hyperglycemia could reach $70 \%$. With elevated $\mathrm{HbA}_{1 \mathrm{c}}$ levels, the contribution rate of BHG gradually increased. Other studies also supported this conclusion [8-15]. Since then, this conclusion was widely quoted in many studies, but the effect of applicable population and therapeutic drugs on this conclusion was often ignored. In fact, whenever checking blood glucose, the setting of normal basal blood glucose threshold and the type of drugs could affect this relationship. Riddle et al. analyzed the data from six randomized controlled trials comparing insulin glargine and other regimens and found that BHG's contribution rate played a major role (76-80\%) at all $\mathrm{HbA}_{1 \mathrm{c}}$ levels $(>7.0 \%)$ before intensive treatment with oral hypoglycemic agents. After intensive treatment, although the $\mathrm{HbA}_{1 \mathrm{c}}$ level declined, BHG's contribution rate decreased more significantly in the patients treated with basal insulin than in the patients treated with other regimens (30.8-41.1\% vs. $63.5-69.9 \%)$ [16-22]. It was evident that different hypoglycemic agents had different effects on the relative contribution rates of BHG and PPHG to overall hyperglycemia.
If one needs to know about a drug's influence on the relative contribution rates, a special study should be designed instead of directly extrapolating this conclusion to this drug. In fact, even for the patients treated with basal insulin, there were ethnic and regional differences in the influence on postprandial hyperglycemia [23].

In the two studies mentioned above $[7,16]$, however, a blood glucose profile of 4 or 7 points during the daytime was selected to calculate glucose area under the curve, which lacked observation of blood glucose during the night. Additionally, during the observation period in these studies, the subjects were given test meals in a test environment, so the obtained blood glucose data did not necessarily reflect "reallife" characteristics.

Recently, a randomized, crossover clinical trial evaluating the effects of insulin lispro mix 25 and 50 (LM25 and LM50) on postprandial glucose excursions in T2DM patients treated with premixed human insulin 70/30 (PHI70/30) showed that LM50 might provide better glycemic excursion control after breakfast and dinner than LM25 [24]. The $\mathrm{HbA}_{1 \mathrm{c}}$ and mean amplitude of glucose excursion (MAGE) values were not different between the three insulin regimens. This study was conducted in a "reallife" environment without changing the subject's diet and activities, using a continuous glucose monitoring (CGM) system to collect the subjects' blood glucose data. The aim of the present post hoc analysis was to investigate the relative contribution rates of BHG and PPHG to overall hyperglycemia in the T2DM patients treated with LM25 and LM50.

\section{METHODS}

\section{Study Design}

The results of this study were from a phase IV, randomized, crossover, open-label, investigatorinitiated clinical trial in Chinese patients with T2DM switching from PHI70/30 to LM25 or LM50. This clinical trial was registered at www. chictr.org.cn (ChiCTR-TTRCC-12002516) [24]. 
This clinical trial consisted of a 2-week screening period, a 2-week lead-in period, and two 16-week crossover treatment periods. After the 2-week screening period, the subjects continued to be treated with PHI70/30 during a 2 -week lead-in period. Then, the subjects were randomly divided into two groups based on a random number table. In the first 16-week stage, one group received LM25 twice daily, the other group received LM50 twice daily. In the second 16-week stage, the two groups exchanged therapeutic regimen. The types and dosages of original oral hypoglycemic agents were not adjusted throughout the trial.

\section{Study Population}

Chinese adult T2DM patients were enrolled to participate in this comparative study on LM25 and LM50 in the Endocrinology Department of Peking Union Medical College Hospital (PUMCH) between November 1, 2013 and May 31,2015 . Before enrollment, the subjects were asked to receive a stable dose of PHI70/30 twice daily (whether combined with oral hypoglycemic agents or not) for at least 90 days.

Major exclusion criteria included patients having liver dysfunction (alanine transaminase or alkaline phosphatase exceeding 2.5 times the upper limit of the normal reference value) or renal insufficiency (serum creatinine $\geq 133 \mu \mathrm{mol} /$ $\mathrm{L}$ for male patients or $\geq 110 \mu \mathrm{mol} / \mathrm{L}$ for female patients), having other diseases or receiving other drugs that may affect blood glucose and hemoglobin, or having an inability to eat normally.

\section{Study Procedures and Treatment}

After enrollment, the subjects continued to be treated with PHI70/30 during a 2-week lead-in period. Then, the subjects were randomly divided into two groups (A and B) based on a random number table.

In the first 16-week stage, the subjects in group A received LM25 twice daily, while the subjects in group B received LM50 twice daily. The first 4 weeks of this stage were the insulin dose titration period. The initial dose of insulin was determined according to the original
PHI70/30 dose. All subjects performed selfmonitoring of blood glucose (SMBG) and got a 4-point blood glucose profile (preprandial blood glucose of three meals plus bedtime blood glucose at least 1 day every week). Follow-up was conducted once every 2 weeks, and insulin dose was adjusted according to the results of SMBG (the adjustment protocol was presented in a previous article [24]). The titration targets of blood glucose were $>3.9$ and $\leq 6.1 \mathrm{mmol} / \mathrm{L}$ before breakfast and dinner. The subsequent 12 weeks of this stage were the steady dose period.

In the second 16-week stage, the two groups exchanged therapeutic regimen. The subjects in group A received LM50 twice daily, while the subjects in group B received LM25 twice daily. Insulin dose was determined according to the dose before crossover, and the insulin dose adjustment method was the same. The types and dosages of original oral hypoglycemic agents were not adjusted throughout the trial.

During the study, it was recommended that the subjects follow China guidelines for diet control and exercise [25]. During the 3 days of CGM at enrollment and the end of each treatment phase, the subjects were given high-carbohydrate test meals on day 1 and high-fat test meals on day 2. They continued their habitual diets on day 3 and recorded their diets and activities. The detailed protocol was presented in a previous article [24].

\section{Outcome Measures}

At enrollment, the subjects' medical history data (age, gender, duration of diabetes, insulin dose, etc.) and anthropometric data (blood pressure, height, weight, waist circumference, etc.) were recorded. $\mathrm{HbA}_{1 \mathrm{c}}$ measurement and $72 \mathrm{~h}$ CGM were performed at enrollment and at the end of each treatment phase. CGM was conducted using the GOLD CGM system (Medtronic, USA). $\mathrm{HbA}_{1 \mathrm{c}}$ was measured by cation-exchange high-performance liquid chromatography (Bio-Rad, USA).

CGM was used to get the glucose area under the curve (AUC). To avoid the effects of diet changes on the blood glucose, only the CGM 
data from day 3 were analyzed in this article. The following definitions were used: AUC with blood glucose $\geq 6.1 \mathrm{mmol} / \mathrm{L}$ was defined as $\mathrm{AUC}_{\mathrm{T}}$ exposed to total hyperglycemia; fasting blood glucose was the average blood glucose $1 \mathrm{~h}$ before breakfast every day; the AUC with blood glucose $\geq$ fasting blood glucose was $A C_{P}$ exposed to postprandial hyperglycemia; the AUC exposed to basal hyperglycemia was $\mathrm{AUC}_{\mathrm{B}}$, $\mathrm{AUC}_{\mathrm{B}}=\mathrm{AUC}_{\mathrm{T}}-\mathrm{AUC}_{\mathrm{P}}$. BHG's contribution rate $=\mathrm{AUC}_{\mathrm{B}} / \mathrm{AUC}_{\mathrm{T}}, \mathrm{PPHG}^{\prime}$ 's contribution rate $=$ $\mathrm{AUC}_{\mathrm{P}} / \mathrm{AUC}_{\mathrm{T}}$. Any negative value of AUC with blood glucose $<6.1 \mathrm{mmol} / \mathrm{L}$ was set to be 0 [16].

\section{Compliance with Ethical Guidelines}

This study was approved by the PUMCH Ethics Committee and followed the ethical standards of the responsible committee on human experimentation (institutional and national) and with the Helsinki Declaration of 1964, as revised in 2013. All patients signed informed consent before enrollment.

\section{Statistical Analysis}

The present study was post hoc in nature. Measurement data were expressed as the mean $\pm \mathrm{SD}$, and count data were expressed as frequency. Paired $t$ test or single factor variance analysis was used to compare measurement data; McNemar's test was used to compare count data. SPSS 22.0 software was used to complete statistical analysis.

\section{RESULTS}

\section{Baseline Characteristics of Subjects}

Eighty-six subjects were enrolled in the study, of which three subjects were excluded because of violation of the study protocol (oral hypoglycemic agents and insulin were discontinued by self) and two subjects requested withdrawal from the trial. Eventually 81 subjects completed
Table 1 Baseline characteristics of the subjects

\begin{tabular}{ll}
\hline Number & 81 \\
Gender (male/female) & $31 / 50$ \\
Age (years) & $59.9 \pm 10.3$ \\
Duration of diabetes (years) & $14.7 \pm 8.4$ \\
BMI (kg/m ${ }^{2}$ ) & $26.3 \pm 4.6$ \\
HbA ${ }_{1 \mathrm{c}}(\%)$ & $7.8 \pm 1.4$ \\
SBP (mmHg) & $122 \pm 13$ \\
DBP (mmHg) & $72 \pm 9$ \\
Weight (kg) & $68.7 \pm 14.8$ \\
Waist circumference (cm) & $87.0 \pm 19.4$ \\
Total daily dose of PHI70/30 (U) & $40.2 \pm 15.4$ \\
Subjects with combined oral hypoglycemic & 90.1 \\
agents (\%) & 68.5 \\
$\quad$ Metformin (\%) & 39.7 \\
Acarbose (\%) & 8.2 \\
Others (\%) & $42.3 \pm 15.3$ \\
Total daily dose of LM25 (U) & $40.8 \pm 15.4$ \\
Total daily dose of LM50 (U) & $840 \pm 468$ \\
Metformin dose (mg/day) & $46 \pm 25$ \\
Acarbose dose (mg/day) & \\
\hline
\end{tabular}

the study. The subjects' baseline characteristics are shown in Table 1 [24].

\section{Comparison of Relative Contribution Rates of BHG and PPHG to Overall Hyperglycemia at Different $\mathrm{HbA}_{1 \mathrm{c}}$ Levels}

According to $\mathrm{HbA}_{1 \mathrm{c}}$ levels at enrollment and at the end of each treatment phase, the $\mathrm{HbA}_{1 \mathrm{c}}$ levels were stratified into five groups from low to high as follows: $<6.5 \%, 6.5$ to $<7.5 \%, 7.5$ to $<8.5 \%, 8.5$ to $<9.5 \%$, and $\geq 9.5 \%$. The contribution rates of BHG and PPHG to overall hyperglycemia at different HbA1c levels are shown in Table 2. 
Table 2 Relative contribution rates of BHG and PPHG to overall hyperglycemia at different $\mathrm{HbA}_{1 \mathrm{c}}$ levels

\begin{tabular}{lllllll}
\hline $\mathbf{H b A}_{\mathbf{1 c}}(\%)$ & $\boldsymbol{n}$ & $\mathbf{A U C}_{\mathbf{T}}$ & $\mathbf{A U C}_{\mathbf{P}}$ & $\mathbf{A U C}_{\mathbf{B}}$ & $\mathbf{A U C}_{\mathbf{P}} / \mathbf{A U C}_{\mathbf{T}}(\%)$ & $\mathbf{A U C}_{\mathbf{B}} / \mathrm{AUC}_{\mathbf{T}}(\%)$ \\
\hline$<6.5$ & 90 & $2.4 \pm 0.7$ & $1.6 \pm 0.5$ & $0.8 \pm 0.4$ & $65.5 \pm 15.3$ & $34.5 \pm 12.2$ \\
6.5 to $<7.5$ & 72 & $3.0 \pm 1.1$ & $1.8 \pm 0.7$ & $1.2 \pm 0.5$ & $61.6 \pm 17.5^{*}$ & $38.4 \pm 16.5^{*}$ \\
7.5 to $<8.5$ & 38 & $3.6 \pm 1.2$ & $1.9 \pm 0.6$ & $1.7 \pm 0.9$ & $53.4 \pm 16.8^{* \#}$ & $46.6 \pm 14.7^{* \#}$ \\
8.5 to $<9.5$ & 31 & $4.3 \pm 1.6$ & $1.9 \pm 1.1$ & $2.4 \pm 1.6$ & $43.5 \pm 16.4^{* \# \wedge}$ & $56.5 \pm 16.9^{* \# \wedge}$ \\
$\geq 9.5$ & 12 & $5.1 \pm 2.0$ & $2.1 \pm 0.7$ & $3.0 \pm 1.1$ & $41.2 \pm 16.3^{* \# \wedge \&}$ & $60.8 \pm 16.9^{* \# \wedge \text { \& }}$ \\
\hline
\end{tabular}

$H b A_{1 c}$ glycosylated hemoglobin, $A U C_{T}$ area under the curve exposed to total hyperglycemia, $A U C_{P}$ area under the curve exposed to postprandial hyperglycemia, $A U C_{B}$ area under the curve exposed to basal hyperglycemia

* vs. $\mathrm{HbA}_{1 \mathrm{c}}<6.5 \%, p<0.05$

\# vs. $\mathrm{HbA}_{1 \mathrm{c}} 6.5$ to $<7.5 \%, p<0.05$

$\wedge^{\wedge}$ vs. $\mathrm{HbA}_{1 \mathrm{c}} 7.5$ to $<8.5 \%, p<0.05$

$\&$ vs. $\mathrm{HbA}_{1 \mathrm{c}} 8.5$ to $<9.5 \%, p<0.05$

Comparison of PPHG's Relative

Contribution Rates Based on Different

Types of Premixed Insulin at Different

$\mathrm{HbA}_{1 \mathrm{c}}$ Levels

According to the type of premixed insulin, the subjects were divided into three groups: PHI70/ 30 group, LM25 group, and LM50 group. Then, the subjects were stratified by the different $\mathrm{HbA}_{1 \mathrm{c}}$ levels at enrollment and at the end of each treatment phase (from low to high as follows: $<6.5 \%, 6.5$ to $<7.5 \%, 7.5$ to $<8.5 \%, 8.5$ to $<9.5 \%$, and $\geq 9.5 \%$ ). PPHG's contribution rates were compared at different $\mathrm{HbA}_{1 \mathrm{c}}$ levels among the three regimens (Table 3 ).

\section{Comparison of Effect of LM25 and LM50 on $\mathrm{HbA}_{1 \mathrm{c}}$ Change at Different $\mathrm{HbA}_{1 \mathrm{c}}$ Levels}

$\mathrm{HbA}_{1 \mathrm{c}} 8.0 \%, 8.5 \%$, and $9.0 \%$ before the subjects were treated with LM25 and LM50 were separately taken as the cutoff points, and the subjects were divided into the less than cutoff point

Table 3 Comparison of relative contribution rates of PPHG to overall hyperglycemia based on different types of premixed insulin at different $\mathrm{HbA}_{1 \mathrm{c}}$ levels

\begin{tabular}{|c|c|c|c|c|c|c|}
\hline \multirow[t]{2}{*}{$\mathrm{HbA}_{1 \mathrm{c}}(\%)$} & \multicolumn{2}{|l|}{ PHI70/30 } & \multicolumn{2}{|l|}{ LM25 } & \multicolumn{2}{|l|}{ LM50 } \\
\hline & $\overline{\mathrm{AUC}_{\mathbf{P}} / \mathrm{AUC}_{\mathbf{T}}(\%)}$ & $n$ & $\overline{\mathrm{AUC}_{\mathbf{P}} / \mathrm{AUC}_{\mathbf{T}}(\%)}$ & $\bar{n}$ & $\overline{\mathrm{AUC}_{\mathbf{P}} / \mathrm{AUC}_{\mathbf{T}}(\%)}$ & $\bar{n}$ \\
\hline$<6.5$ & $68.9 \pm 13.8$ & 26 & $67.5 \pm 14.6$ & 33 & $60.5 \pm 15.6^{* \#}$ & $\overline{31}$ \\
\hline 6.5 to $<7.5$ & $65.7 \pm 16.5$ & 25 & $66.5 \pm 17.8$ & 23 & $52.6 \pm 15.6^{* \#}$ & 24 \\
\hline 7.5 to $<8.5$ & $55.9 \pm 18.5$ & 15 & $55.0 \pm 18.0$ & 11 & $48.8 \pm 17.2$ & 12 \\
\hline 8.5 to $<9.5$ & $46.2 \pm 16.6$ & 10 & $46.7 \pm 16.3$ & 11 & $37.3 \pm 17.8$ & 10 \\
\hline$\geq 9.5$ & $43.2 \pm 16.7$ & 5 & $42.4 \pm 16.3$ & 3 & $37.8 \pm 15.8$ & 4 \\
\hline Total & & 81 & & 81 & & 81 \\
\hline
\end{tabular}

$H b A_{\text {Ic }}$ glycosylated hemoglobin, $P H I 70 / 30$ premixed human insulin 70/30, LM25 insulin lispro mix 25, LM50 insulin lispro mix 50, $A U C_{P}$ area under the curve exposed to postprandial hyperglycemia, $A U C_{T}$ area under the curve exposed to total hyperglycemia

* vs. PHI70/30, $p<0.05$

\# vs. LM25, $p<0.05$ 
Table 4 Comparison of effect of LM25 and LM50 on HbA 1 change at different HbA $1 \mathrm{c}$ levels

\begin{tabular}{|c|c|c|c|c|c|c|c|c|c|}
\hline \multicolumn{2}{|c|}{$\mathrm{HbA}_{1 \mathrm{c}}(\%)$} & \multicolumn{4}{|l|}{ LM25 } & \multicolumn{4}{|l|}{ LM50 } \\
\hline $\begin{array}{l}\text { Cutoff } \\
\text { point }\end{array}$ & $\overline{\text { Subgroup }}$ & $\begin{array}{l}\text { Before } \\
\text { treatment }\end{array}$ & $\begin{array}{l}\text { After } \\
\text { treatment }\end{array}$ & $\Delta \mathrm{HbA}_{1 \mathrm{c}}(\%)$ & $n$ & $\begin{array}{l}\text { Before } \\
\text { treatment }\end{array}$ & $\begin{array}{l}\text { After } \\
\text { treatment }\end{array}$ & $\Delta \mathrm{HbA}_{1 \mathrm{c}}(\%)$ & $\bar{n}$ \\
\hline \multirow[t]{2}{*}{8.0} & $<8.0$ & $7.4 \pm 0.8$ & $7.2 \pm 0.8$ & $-0.2 \pm 0.1$ & 57 & $7.3 \pm 0.8$ & $7.1 \pm 0.7$ & $-0.2 \pm 0.2$ & 58 \\
\hline & $\geq 8.0$ & $8.8 \pm 1.0$ & $8.5 \pm 0.9$ & $-0.3 \pm 0.2$ & 24 & $8.8 \pm 0.9$ & $8.6 \pm 0.8$ & $-0.2 \pm 0.1$ & 23 \\
\hline \multirow[t]{2}{*}{8.5} & $<8.5$ & $7.5 \pm 0.9$ & $7.4 \pm 0.9$ & $-0.1 \pm 0.1$ & 66 & $7.5 \pm 1.0$ & $7.1 \pm 0.9$ & $-0.4 \pm 0.1$ & 66 \\
\hline & $\geq 8.5$ & $9.3 \pm 1.0$ & $8.7 \pm 1.0$ & $-0.6 \pm 0.1$ & 15 & $8.8 \pm 1.0$ & $9.1 \pm 1.1$ & $0.3 \pm 0.1^{*}$ & 15 \\
\hline \multirow[t]{2}{*}{9.0} & $<9.0$ & $7.6 \pm 1.0$ & $7.5 \pm 0.9$ & $-0.1 \pm 0.1$ & 72 & $7.5 \pm 0.9$ & $7.3 \pm 0.9$ & $-0.2 \pm 0.1$ & 71 \\
\hline & $\geq 9.0$ & $9.4 \pm 1.1$ & $8.8 \pm 1.0$ & $-0.6 \pm 0.2$ & 9 & $9.2 \pm 1.0$ & $8.9 \pm 1.0$ & $-0.3 \pm 0.1$ & 10 \\
\hline
\end{tabular}

$H b A_{1 c}$ glycosylated hemoglobin, LM25 insulin lispro mix 25, LMSO insulin lispro mix 50, $\triangle H b A_{1 c} \mathrm{HbA}_{1 \mathrm{c}}$ after treatment $-\mathrm{HbA}_{1 \mathrm{c}}$ before treatment

* vs. LM25, $p<0.05$

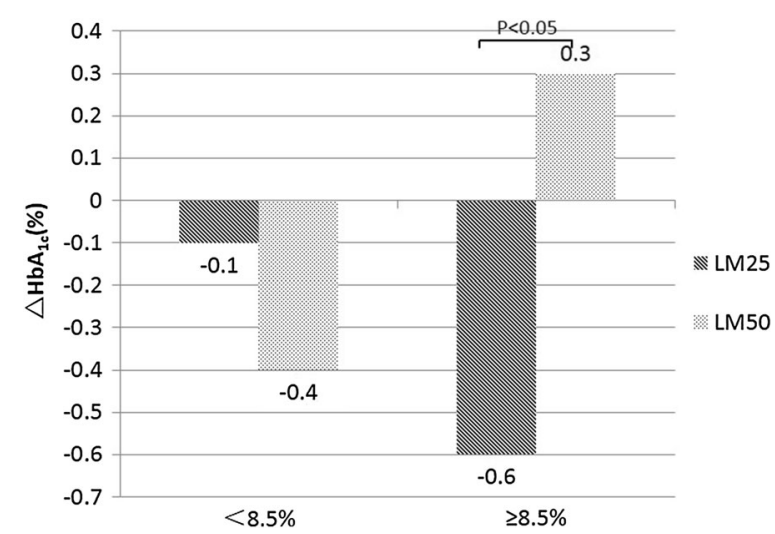

Fig. 1 Comparison of $\Delta \mathrm{HbA}_{1 \mathrm{c}}$ between $\mathrm{HbA}_{1 \mathrm{c}}<8.5 \%$ subgroup and $\mathrm{HbA}_{1 \mathrm{c}} \geq 8.5 \%$ subgroup before and after LM25 and LM50 treatment

subgroup and at least the cutoff point subgroup to compare the changes in $\mathrm{HbA}_{1 \mathrm{c}}$ of the two subgroups before and after treatment $\left(\Delta \mathrm{HbA}_{1 \mathrm{c}}=\mathrm{HbA}_{1 \mathrm{c}}\right.$ after treatment $-\mathrm{HbA}_{1 \mathrm{c}}$ before treatment). It was found that if $8.5 \%$ was taken as the cutoff point, LM25 was more effective than LM50 in reducing $\mathrm{HbA}_{1 \mathrm{c}}$ in the subgroup with baseline $\mathrm{HbA}_{1 \mathrm{c}} \geq 8.5 \%\left(\Delta \mathrm{HbA}_{1 \mathrm{c}}\right.$ LM25 vs. LM50 $-0.6 \pm 0.1 \%$ vs. $0.3 \pm 0.1 \%$, $p<0.05$ ). If other $\mathrm{HbA}_{1 \mathrm{c}}$ cutoff points were used to divide the groups, the difference in $\Delta \mathrm{HbA}_{1 \mathrm{c}}$ between the two subgroups was not significant (Table 4 and Fig. 1).

\section{DISCUSSION}

The present post hoc analysis showed that for the patients with type 2 diabetes treated with premixed human insulin or insulin analogues (in combination with oral hypoglycemic agents or not), postprandial hyperglycemia played a major role in the subgroup of patients with mild to moderate hyperglycemia $\left(\mathrm{HbA}_{1 \mathrm{c}}<8.5 \%\right)$. However, with further deterioration in blood glucose, fasting hyperglycemia became the major contributor to overall hyperglycemia. These findings were consistent with Monnier's study, although this study was different from Monnier's study in study subjects, types of hypoglycemic agents, and blood glucose monitoring methods [7]. Therefore, the conclusions of Monnier's study were applicable to this study population treated with premixed human insulin or insulin analogues. Compared with Monnier's study, this study had several advantages: CGM was used as the blood glucose evaluation method for all patients in the study, which could fully reflect the changes in blood glucose during the day and night, so this study could get more information about blood glucose. In Monnier's study, the subjects received a test meal in a test environment; in contrast, this study collected day 3's CGM data without changing the subjects' diet and activities, which 
may better reflect the real relationship between short-term changes in blood glucose and $\mathrm{HbA}_{1 \mathrm{c}}$.

The conclusions of this study were not consistent with those of Riddle et al.'s study [16]. Their study concluded that BHG's contribution rate played a major role regardless of $\mathrm{HbA}_{1 \mathrm{c}}$ levels $(>7 \%)$ before intensive treatment. It was considered that the difference was due to the normal blood glucose cutoff point of $6.1 \mathrm{mmol} /$ L selected in this study (based on the recommendations of China Guideline for Type 2 Diabetes Mellitus) [26]. In Riddle's study, the normal blood glucose cutoff point was $5.6 \mathrm{mmol} / \mathrm{L}$, so the $\mathrm{AUC}_{\mathrm{B}}$ in this study was smaller than that in Riddle's study. Besides, the study population included in this study also involved subjects with $\mathrm{HbA}_{1 \mathrm{c}}<6.5 \%$. In Riddle's study, all subjects' $\mathrm{HbA}_{1 \mathrm{c}}$ were $>7.0 \%$, so the contribution of PPHG might be higher in the subjects with mild hyperglycemia. Moreover, Riddle et al.'s study population comprised subjects with poor glycemic control treated with oral hypoglycemic agents, who had higher basal hyperglycemia generally than our study population treated with premixed insulin.

At present, there are several clinical trials directly comparing the effects of LM25 and LM50 on fasting hyperglycemia, postprandial hyperglycemia, and $\mathrm{HbA}_{1 \mathrm{c}}$ [27-31]. Our study and the above studies all showed that LM50 was more effective than LM25 in reducing postprandial blood glucose after the morning and evening meals, reducing prandial blood glucose excursion or stabilizing blood glucose excursion during the daytime [24]. Similarly, in the present post hoc analysis, we also observed that the contribution rates of PPHG to overall hyperglycemia in LM50 were lower than those in LM25 at $\mathrm{HbA}_{1 \mathrm{c}}$ levels $<7.5 \% \quad\left(\mathrm{AUC}_{\mathrm{P}} / \mathrm{AUC}_{\mathrm{T}}\right.$ LM50 vs. LM25 52.6-60.5\% vs. 66.5-67.5\%, $p<0.05)$. This may be because the contribution rates of PPHG played a major role (61.6-65.5\%) and LM50 reduced PPHG more significantly than LM25 in this $\mathrm{HbA}_{1 \mathrm{c}}$ interval.

In these studies, there was no significant difference in the effect of LM25 and LM50 on $\mathrm{HbA}_{1 \mathrm{c}}$. But the CLASSIFY study showed that more subjects in the LM50 group than in the LM25 group achieved $\mathrm{HbA}_{1 \mathrm{c}}$ targets. The results of further stratified analysis were also different. Our study showed that LM25 was more effective than LM50 in reducing $\mathrm{HbA}_{1 \mathrm{c}}$ in the subgroup with baseline $\mathrm{HbA}_{1 \mathrm{c}} \geq 8.5 \% \quad\left(\Delta \mathrm{HbA}_{1 \mathrm{c}} \quad \mathrm{LM} 25 \quad\right.$ vs. LM50 $-0.6 \pm 0.1 \%$ vs. $0.3 \pm 0.1 \%, p<0.05)$. According to the theory of Monnier and the results of our study, it was not difficult to understand that in the subgroup with higher $\mathrm{HbA}_{1 \mathrm{c}}$ levels ( $\geq 8.5 \%$ ), low-ratio premixed insulin LM25 (containing 25\% rapid-acting component and $75 \%$ intermediate-acting component) with the main goal of reducing basal hyperglycemia was more effective than mid-ratio premixed insulin LM50 (containing 50\% rapid-acting component and $50 \%$ intermediate-acting component) with the main goal of reducing postprandial hyperglycemia in reducing $\mathrm{HbA}_{1 \mathrm{c}}$ levels. But in the subgroup with lower baseline $\mathrm{HbA}_{1 \mathrm{c}}$ levels $(<8.5 \%)$, LM50 was similar to LM25 in reducing $\mathrm{HbA}_{1 \mathrm{c}}\left(\Delta \mathrm{HbA}_{1 \mathrm{c}} \mathrm{LM} 50\right.$ vs. LM25 $-0.4 \pm 0.1 \%$ vs. $-0.1 \pm 0.1 \%, p>0.05)$. However, the series of CLASSIFY studies indicated that LM50 was more effective than $\mathrm{LM} 25$ in reducing $\mathrm{HbA}_{1 \mathrm{c}}$ in subjects with baseline $\mathrm{HbA}_{1 \mathrm{c}}$ levels greater than the median levels. The reason for this difference was not very clear. It was supposed to be related to the different types of baseline drugs and $\mathrm{HbA}_{1 \mathrm{c}}$ levels in the study populations. It also suggested that we should pay attention to the characteristics of applicable populations and not extrapolate blindly when applying the findings of a study.

A limitation of this study is that the sample size is relatively small. Especially after $\mathrm{HbA}_{1 \mathrm{c}}$ was stratified, the number of some subgroups was small, which may affect the test efficiency. In addition, the crossover design may also have an impact on the outcome of the treatment at a later stage. CGM data of only 1 day in each phase were analyzed, so that they provided limited information. However, this study still helps us to understand the effects of fasting and postprandial blood glucose on overall blood glucose in T2DM patients with premixed insulin analogue.

\section{CONCLUSION}

Our study found that for patients with type 2 diabetes treated with premixed insulin analogue combined with oral hypoglycemic agents or 
not, postprandial hyperglycemia played a major role in the subgroup patients with mild to moderate hyperglycemia $\left(\mathrm{HbA}_{1 \mathrm{c}}<8.5 \%\right)$. With further deterioration in blood glucose, fasting hyperglycemia became the major contributor to overall hyperglycemia. LM25 was more effective than LM50 in reducing HbA1c in the subgroup with baseline $\mathrm{HbA1c} \geq 8.5 \%$.

\section{ACKNOWLEDGEMENTS}

We thank the participants of the study.

Funding. This study was funded by Lilly Suzhou Pharmaceutical Co., Ltd. (Shanghai Branch, China) and the National Key Program of Clinical Science of China (WBYZ 2011-873). The article processing charges were funded by the authors. All authors had full access to all of the data in this study and take complete responsibility for the integrity of the data and accuracy of the data analysis.

Authorship. All named authors meet the International Committee of Medical Journal Editors (ICMJE) criteria for authorship for this manuscript, take responsibility for the integrity of the work as a whole, and have given final approval to the version to be published. Prof. Y. Li is the guarantor for the study and takes full responsibility for the integrity of the work as a whole, from inception to published article.

Disclosures. Wei Li, Hongmei Li, Fan Ping, Lingling $\mathrm{Xu}$, Huabing Zhang, Yaxiu Dong, Qi Sun and Yuxiu Li have nothing to disclose.

Compliance with Ethics Guidelines. This study was approved by the PUMCH Ethics Committee and followed the ethical standards of the responsible committee on human experimentation (institutional and national) and with the Helsinki Declaration of 1964, as revised in 2013. All patients signed informed consent before enrollment.

Data Availability. The datasets generated and/or analyzed during the current study are available from the corresponding author on reasonable request.

Open Access. This article is distributed under the terms of the Creative Commons Attribution-NonCommercial 4.0 International License (http://creativecommons.org/licenses/ by-nc/4.0/), which permits any noncommercial use, distribution, and reproduction in any medium, provided you give appropriate credit to the original author(s) and the source, provide a link to the Creative Commons license, and indicate if changes were made.

\section{REFERENCES}

1. The Diabetes Control and Complications Trial Research Group. The effect of intensive treatment of diabetes on the development and progression of long-term complications in insulin-dependent diabetes mellitus. N Engl J Med. 1993;329:977-86.

2. Writing Team for the Diabetes Control and Complications Trial/Epidemiology of Diabetes Interventions and Complications Research Group. Effect of intensive therapy on the microvascular complications of type 1 diabetes mellitus. JAMA. 2002;287:2563-9.

3. Diabetes Control and Complications Trial (DCCT)/ Epidemiology of Diabetes Interventions and Complications (EDIC) Research Group, Lachin JM, White $\mathrm{NH}$, et al. Effect of intensive diabetes therapy on the progression of diabetic retinopathy in patients with type 1 diabetes: 18 years of follow-up in the DCCT/EDIC. Diabetes. 2015;64:631-42.

4. UK Prospective Diabetes Study (UKPDS) Group. Intensive blood-glucose control with sulphonylureas or insulin compared with conventional treatment and risk of complications in patients with type 2 diabetes (UKPDS 33). Lancet. 1998;352:837-53.

5. Holman RR, Paul SK, Bethel MA, et al. 10-year follow-up of intensive glucose control in type 2 diabetes. N Engl J Med. 2008;359:1577-89.

6. Davis TM, Coleman RL, Holman RR. Ethnicity and long-term vascular outcomes in type 2 diabetes: a prospective observational study (UKPDS 83). Diabet Med. 2014;31:200-7.

7. Monnier L, Lapinski H, Colette C. Contributions of fasting and postprandial plasma glucose increments to the overall diurnal hyperglycemia of type 2 
diabetic patients: variations with increasing levels of $\mathrm{HbA}(1 \mathrm{c})$. Diabetes Care. 2003;26:881-5.

8. Peter R, Luzio SD, Dunseath G, et al. Relationship between HbA1c and indices of glucose tolerance derived from a standardized meal test in newly diagnosed treatment naive subjects with type 2 diabetes. Diabet Med. 2006;23:990-5.

9. Monnier L, Colette C, Dunseath GJ, et al. The loss of postprandial glycemic control precedes stepwise deterioration of fasting with worsening diabetes. Diabetes Care. 2007;30:263-9.

10. Woerle HJ, Neumann C, Zschau S, et al. Impact of fasting and postprandial glycemia on overall glycemic control in type 2 diabetes Importance of postprandial glycemia to achieve target HbA1c levels. Diabetes Res Clin Pract. 2007;77:280-5.

11. Peter R, Dunseath G, Luzio SD, et al. Relative and absolute contributions of postprandial and fasting plasma glucose to daytime hyperglycaemia and $\mathrm{HbA}(1 \mathrm{c})$ in subjects with type 2 diabetes. Diabet Med. 2009;26:974-80.

12. Kikuchi K, Nezu U, Shirakawa J, et al. Correlations of fasting and postprandial blood glucose increments to the overall diurnal hyperglycemic status in type 2 diabetic patients: variations with levels of HbA1c. Endocr J. 2010;57:259-66.

13. Schernthaner G, Guerci B, Gallwitz B, et al. Impact of postprandial and fasting glucose concentrations on $\mathrm{HbA} 1 \mathrm{c}$ in patients with type 2 diabetes. Diabetes Metab. 2010;36:389-94.

14. Wang JS, Tu ST, Lee IT, et al. Contribution of postprandial glucose to excess hyperglycaemia in Asian type 2 diabetic patients using continuous glucose monitoring. Diabetes Metab Res Rev. 2011;27:79-84.

15. Monnier L, Colette C, Dejager S, et al. Residual dysglycemia when at target $\mathrm{HbA}(1 \mathrm{c})$ of $7 \%$ $(53 \mathrm{mmol} / \mathrm{mol})$ in persons with type 2 diabetes. Diabetes Res Clin Pract. 2014;104:370-5.

16. Riddle M, Umpierrez G, DiGenio A, et al. Contributions of basal and postprandial hyperglycemia over a wide range of A1C levels before and after treatment intensification in type 2 diabetes. Diabetes Care. 2011;34:2508-14.

17. Riddle MC, Rosenstock J, Gerich J. The treat-totarget trial: randomized addition of glargine or human NPH insulin to oral therapy of type 2 diabetic patients. Diabetes Care. 2003;26:3080-6.

18. Janka HU, Plewe G, Riddle MC, et al. Comparison of basal insulin added to oral agents versus twice-daily premixed insulin as initial insulin therapy for type 2 diabetes. Diabetes Care. 2005;28:254-9.

19. Gerstein HC, Yale JF, Harris SB, et al. A randomized trial of adding insulin glargine vs. avoidance of insulin in people with type 2 diabetes on either no oral glucose-lowering agents or submaximal doses of metformin and/or sulphonylureas. The Canadian INSIGHT (Implementing New Strategies with Insulin Glargine for Hyperglycaemia Treatment) Study. Diabet Med. 2006;23:736-42.

20. Standl E, Maxeiner S, Raptis S. Once-daily insulin glargine administration in the morning compared to bedtime in combination with morning glimepiride in patients with type 2 diabetes: an assessment of treatment flexibility. Horm Metab Res. 2006;38:172-7.

21. Yki-Jarvinen H, Kauppinen-Makelin R, Tiikkainen $\mathrm{M}$, et al. Insulin glargine or NPH combined with metformin in type 2 diabetes: the LANMET study. Diabetologia. 2006;49:442-51.

22. Bretzel RG, Nuber U, Landgraf W, et al. Once-daily basal insulin glargine versus thrice-daily prandial insulin lispro in people with type 2 diabetes on oral hypoglycaemic agents (APOLLO): an open randomised controlled trial. Lancet. 2008;371:1073-84.

23. Shaefer C, Reid T, DiGenio A, et al. Patterns of postprandial hyperglycemia after basal insulin therapy: individual and regional differences. Diabetes Metab Res Rev. 2014. https://doi.org/10.1002/ dmrr.2606.

24. Li W, Ping F, Xu L, et al. Effects of insulin lispro mix 25 and insulin lispro mix 50 on postprandial glucose excursion in patients with type 2 diabetes: a prospective, open-label, randomized clinical trial. Diabetes Ther. 2018;9:699-711.

25. Chinese Diabetes Society, China Medicine Doctor Association Nutrition Doctor Specialized Committee. China medical nutrition therapy guideline for diabetes. Chin J Diabetes Mellitus. 2013;2015(7):73-88 (Chinese).

26. Chinese Diabetes Society. China guideline for type 2 diabetes mellitus (2013 Edition). Chin J Endocrinol Metab. 2014;30:893-942. https://doi.org/10. 3760/cma.j.issn.1000-6699.2014.10.020 (Chinese).

27. Tanaka M, Ishii H. Pre-mixed rapid-acting insulin $50 / 50$ analogue twice daily is useful not only for controlling post-prandial blood glucose, but also for stabilizing the diurnal variation of blood glucose levels: switching from pre-mixed insulin $70 / 30$ or $75 / 25$ to pre-mixed insulin 50/50. J Int Med Res. 2010;38:674-80. 
28. Nishimura R, Tsujino D, Taki K, et al. Continuous glucose monitoring with Humalog Mix 25 versus Humalog Mix 50, twice daily: a comparative pilot study -results from the Jikei-EValuation of insulin Lispro mixture on pharmacodynamics and glycemic VariancE (J-EVOLVE) study. Cardiovasc Diabetol. 2010;9:16. https://doi.org/10.1186/14752840-9-16.

29. Watada H, Su Q, Li PF, Iwamoto N, Qian L, Yang WY. Comparison of insulin lispro mix 25 with insulin lispro mix 50 as an insulin starter in Asian patients with type 2 diabetes: a phase 4 , open-label, randomized trial (CLASSIFY study). Diabetes Metab Res Rev. 2017;33:e2816.
30. Su Q, Liu C, Zheng H, et al. Comparison of insulin lispro mix 25 with insulin lispro mix 50 as insulin starter in Chinese patients with type 2 diabetes mellitus (CLASSIFY study): subgroup analysis of a phase 4 open-label randomized trial. J Diabetes. 2017;9:575-85.

31. Su Q, Liu J, Li P, Qian L, Yang W. Relative contribution of fasting and postprandial blood glucose in overall glycemic control: post hoc analysis of a phase IV randomized trial. Diabetes Ther. 2018. https://doi.org/10.1007/s13300-018-0403-7. 